\title{
To Study the Effects of Shenqifuzheng Injection (SFI) on Micro-RNAs in Human Dendritic Cells
}

Yuwh H*, Sze WH, Yip DMY, Cho SP, Boost WCS, Zhang MV, Fan Z, Ng K, Ng MCH, Yeung JWC, Hung A, H WH, Chong GSL and Lee RLP

Faculty of Health and Social Sciences, The Hong Kong Polytechnic University, Hong Kong

\begin{abstract}
Method: This study used a 2-step approach. By first we were using a robust DC cell line to identify those miRNA that might be involved in DC activation; then followed by confirmation using monocyte-derived DC which was closer to real-life physiological contexts. THP-1 cell, a human monocytic leukemia cell line, has previously been shown to be useful for the in vitro study of DC. In this study, we identified a number of potential miRNA candidates in the DCs activated by SFI using MicroRNAArray of 381 known human miRNAs (Sanger miR Base, version 14). miR-22, miR-107 and miR-200a were then selected from the over 30 potential miRNA candidates with relative expression fold change $>2$ indentified in accordance to the computational evidences retrieved in the annotation database pipeline miR Base. These three miRNAs were further examined for any change in expression in a confirmatory study using the monocyte-derived DCs (MDDC) from peripheral blood mononuclear cells of healthy normal subjects $(n=7)$ through the quantification by Real Time RT-PCR quantification. These results were correlated with the expression levels of HLA-DR and CD80 on the MDDCs using flow cytometry.
\end{abstract}

Results: The confirmatory results did not show any consistent up-regulation in the expressions of miR22, miR107 and miR-200a due to SFI across the seven MDDC samples. In addition, test results on MDDCs upon treatment of SFI in two different dilutions of $1 / 20$ and $1 / 40$ respectively for 24 hours incubation also demonstrated insignificant expression changes of HLA-DR and CD80 ( $>>0.05)$. Furthermore, the correlation of the expression three mi-RNA and the surface expression levels of HLA-DR and CD80 were not statistically significant for MDDCs with treatment of SFI diluted in 1/20 for 1 hour, SFI diluted in 1/20 for 4 hours, and SFI diluted in $1 / 40$ for 1 hour $(p>0.05)$.

Conclusions: Increased expression of miRNA of miR-22, miR-107 and miR-200a were found only in the cell line of THP-1, but failed to be shown consistently in the MDDCs from seven human peripheral blood samples. Thus whether these three mi-RNAs were really important epigenetic regulators during the process of SFI on DC remain unknown; and will require more future investigations by using multiple authentic DC sources.

\section{Introduction}

Shenqifuzheng Injection (SFI) has been used in China as a clinically-useful traditional Chinese medicine (TCM) in cancer management. Our study was to explore if SFI would enhance the immune-modulatory effects of the professional antigen presenting cells - dendritic cells (DCs). DCs are significant in natural immunity against cancer. In this study, expression levels of HLA-DR and CD80 on DCs induced by SFI were measured by flow cytometric analysis. It had been reported that microRNA (miRNA) was central in the epigenetic control of gene expression at the cellular post-translational level. We hypothesized that by understanding the changes of miRNA profile expression during the treatment of DCs by the SFI, we might identify a few critical miRNA that would be instrumental in the DC activation and in turn leading to effective $\mathrm{T}$ cell priming.

Use of the TCM agent - SFI achieves a clinical anti-cancer effect that provides specific, durable and non-toxic therapy. This study aids in the understanding of the immune regulatory capacity of SFI on miRNA in human DCs. Measurement of miRNA can provide potentially very useful information for subsequent research especially concerning their epigenetic regulatory properties. The study also aids in the identification of possible reliable markers associated with immuno-stimulatory DCs, which may allow better diagnosis and improved prognosis for patients as part of the managed healthcare system. In addition, this study may provide essential insights into the understanding of epigenetic variations of the immune system by manipulation of miRNAs in human DCs for the future development of novel strategies for controlling graft rejection $[1,2]$. This in turn may enhance the synergetic effect of combining Western drugs with TCM components through the approach of systems medicine in cancer therapy: understanding life, improving health [2,3] and possibly reducing the gap between the developed and underdeveloped nations in providing effective treatments.

\section{Methods and Materials}

\section{Aims}

The aim of the study was to explore the possible effects of SFI on miRNAs in human DCs through the evaluation of the expression changes of the target miRNAs in the monocyte-derived DCs treated with SFI.

\section{The hypothesis of the study}

The SFI would induce effects on some of the miRNAs in human DC.

\section{Study design}

In order to answer the research question exploring the possible effects of SFI on miRNAs in human DCs, the miRNAs expression

${ }^{*}$ Corresponding author: Yuwh H, Faculty of Health and Social Sciences, The Hong Kong Polytechnic University, Hong Kong; E-mail: hong.yuwh@connect.polyu.hk

Received July 24, 2013; Accepted January 25, 2014; Published January 29 2014

Citation: Yuwh H, Sze WH, Yip DMY, Cho SP, Boost WCS, et al. (2014) To Study the Effects of Shenqifuzheng Injection (SFI) on Micro-RNAs in Human Dendritic Cells. J Data Mining Genomics Proteomics 5: 149. doi:10.4172/2153-0602.1000149

Copyright: $\odot 2014$ Yuwh H, et al. This is an open-access article distributed unde the terms of the Creative Commons Attribution License, which permits unrestricted use, distribution, and reproduction in any medium, provided the original author and source are credited. 
changes in human monocyte-derived DCs were evaluated after a few miRNAs had been identified with the use of SFI treated THP-1 cells in a miRNA array study (TaqMan ${ }^{\circledR}$ Array Human MicroRNA A+B Card Set v3 of Part Number 4444913, Applied Biosystems). Research hypothesis would expect the presence of effects of the SFI on some identified miRNAs in human DCs. Samples of monocyte-derived DCs from a number of normal subjects were used to verify the presence of expression changes of the identified miRNAs under the effects of SFI by real time RT-PCR (Applied Biosystems 7900HT).

\section{Inclusion and exclusion criteria}

Samples of the male or female subjects being the normal blood donors from Hong Kong Red Cross Blood Transfusion Service were included in the study. Donors with signs of inflammation such as respiratory tract infection would be excluded.

\section{Differential expression of miRNAs by the miRNA array study}

The differential expressions of miRNAs in THP-1 treated cells were analysed with the signatures of miRNA expressions down-regulated or up-regulated. The MDDCs from normal subjects would then be used to determine if the differential expressions of these miRNAs were reproducible as those seen in THP-1 cells.

\section{Biological half-life of miRNAs}

Most of the miRNAs have a long half-life (t1/2) which would be greater than 24 hours. It was not observed there were fast degrading miRNAs (t1/2 $<8$ hours) http://www.broadinstitute.org/files/shared/ diversity/summerprogram/2008/dmcdonald_presentation.ppt\#281, 18, What Does This All Mean? As on 21 May 2011).

The panel of miRNAs with extremely stability included Let-7i \& miR155 (t1/2 up to $99 \& 101$ hours respectively); miR125b (t1/2 up to 225 hours or 9 days) and miR208 (t1/2 up to 12 days). miR21 and miR146a were even more stable than miR155. The stability of miRNAs showed 10 times more stable than that of mRNAs. The persistence of the miRNAs was related to the biological functions [4-6].

\section{MDDC preparation}

Units of buffy coat were obtained from some healthy donor blood units. Monocytes were purified by positive selection using anti-CD14 conjugated with magnetic microbeads, Human (Miltenyi Bio-tec, CA 95602 USA). DCs were generated by culturing monocytes for 6 days in RPMI 1640, 10\% FCS supplemented with Recombinant Human GMCSF (100ng/mL) and Recombinant Human IL-4 (50ng/mL) (R\&D Systems, MN55413 USA). The above medium would be changed once after 3 days cultivation in the culture period of total 6 days. On the sixth day of incubation, the portions of mature DCs were gently aspirated, treated with SFI for the designated time points and then harvested for total RNA extraction process which would be further confirmed for the miRNA expression in human MDDCs. Another portions of mature DCs treated with SFI overnight were analyzed by flow cytometry to access the phenotypes of matured and SFI treated DCs.

\section{Monocyte purification and CD14 microbeads application}

Firstly, the CD14+ cells in the PBMC sample were magnetically labeled with CD14 MicroBeads. Then, the cell suspension was applied onto a MACS syringe column, which was placed in the magnetic field of a MACS Separator, held in place by a stand. The magnetically labeled CD14+ cells were retained within the column. The unlabeled cell fractions depleted of CD14+ cells ran through and were collected as the negative fraction. After removal of the column from the magnetic field, the magnetically retained CD14+ cells were eluted as the positively selected cell fraction.

\section{GM-CSF and IL-4 treatment}

DCs of myeloid origin are derived from hematopoietic progenitor cells with myeloid differentiation potential. Immunostimulatory DCs can be generated from monocytes. The differentiation process without proliferation was induced in culture by granulocyte-macrophage colony-stimulating factor (GM-CSF) and IL-4 $[7,8]$.

Purified monocytes were then cultured in 24-well plates in RPMI 1640 medium supplemented with 10 percent fetal calf serum, $1 \%$ penicillin, $1 \%$ L-glutamine, recombinant GM-CSF $(100 \mathrm{ng} / \mathrm{mL})(\mathrm{R} \& \mathrm{D}$ Systems, USA) and IL-4 (50 ng/mL) (R\&D systems, USA). The above medium was changed once every 3 days over the culture period of 6 days.

\section{MDDC harvest and treatment with SFI}

MDDCs $\left(1 \times 10^{5} / \mathrm{mL}\right)$ were treated with SFI $(1 / 20 \& 1 / 40)$ and without SFI in RPMI_1640 on day 6. The SFI treated MDDCs for short time points were then further processed for RNA extraction and the extracted RNA samples were kept at $-80^{\circ} \mathrm{C}$ which was further pending for real time RT-PCR on selected target miRNAs. The other two portions of MDDCs with and without SFI treatment had been cultured for 24 hours before they were analysed with flow cytometry for cell surface markers.

\section{Flow cytometric analysis of the harvested cells}

Successful derivation of monocyte into MDDC could be verified by the expression of CD83 which is the maturation marker for the differential monocytes. CD80 and HLA-DR are both immunocostimulatory markers on the MDDC acting as essential specific markers for DCs [7-10]. Expression changes of HLA-DR and CD80 on cell surface of harvested MDDCs were analysed with corresponding monoclonal antibodies against their isotypic controls. Flow cytometry acquisition and analysis were performed with cytometric analyser CXP FC 500 (Beckman Coulter).

\section{Annotation for TLR-4 related miRNAs at data repositories}

Annotation of miRNAs involved in the interplay was performed with over 30 genes in TLR-4 signaling pathways which included MyD88 dependent and MyD88 independent, and MHCII of antigen processing and presentation pathways. The interplaying genes included TIRAP, MyD88, IRAK4, IRAK1, TRAF6, TAB1, TAB2, TAK1, IKK, NFkB, MKK, p38, JNK, AP1, TNF $\alpha$, IL1 $\beta$, IL6, IL12, IL-8, RANTES, TRAM, TRIF, TRAF3, IKKe, TBK1, IRF3, IRF7, CD40, CD80, CD86, IFN- $\alpha$, IFN- $\beta$, IP10, MIG, I-TAC, GILT, AEP, CTSB, Ii, MHCII, SLIP, CTSB/ L/S, CLIP, HLA-DM, CD4, CIITA, RFX, CREB, NFY and MHCI (Kyoto Encyclopedia of Genes and Genome available at http://www.genome. $\mathrm{jp} / \mathrm{kegg} /$ ).

A few miRNAs with over expression chosen for the miRNA array study were matched with those clustered in the annotation findings for the TLR- 4 related ones.

\section{Computational miRNAs in association with co-stimulatory CD80}

As the aim was to correlate well-expressed miRNAs with the costimulatory effects of treatment with SFI on the TLR-4 receptor, the evidence of links with the cell marker gene CD80 were then focused on the related pipeline searches and a few miRNAs were identified. These 
Citation: Yuwh H, Sze WH, Yip DMY, Cho SP, Boost WCS, et al. (2014) To Study the Effects of Shenqifuzheng Injection (SFI) on Micro-RNAs in Human Dendritic Cells. J Data Mining Genomics Proteomics 5: 149. doi:10.4172/2153-0602.1000149

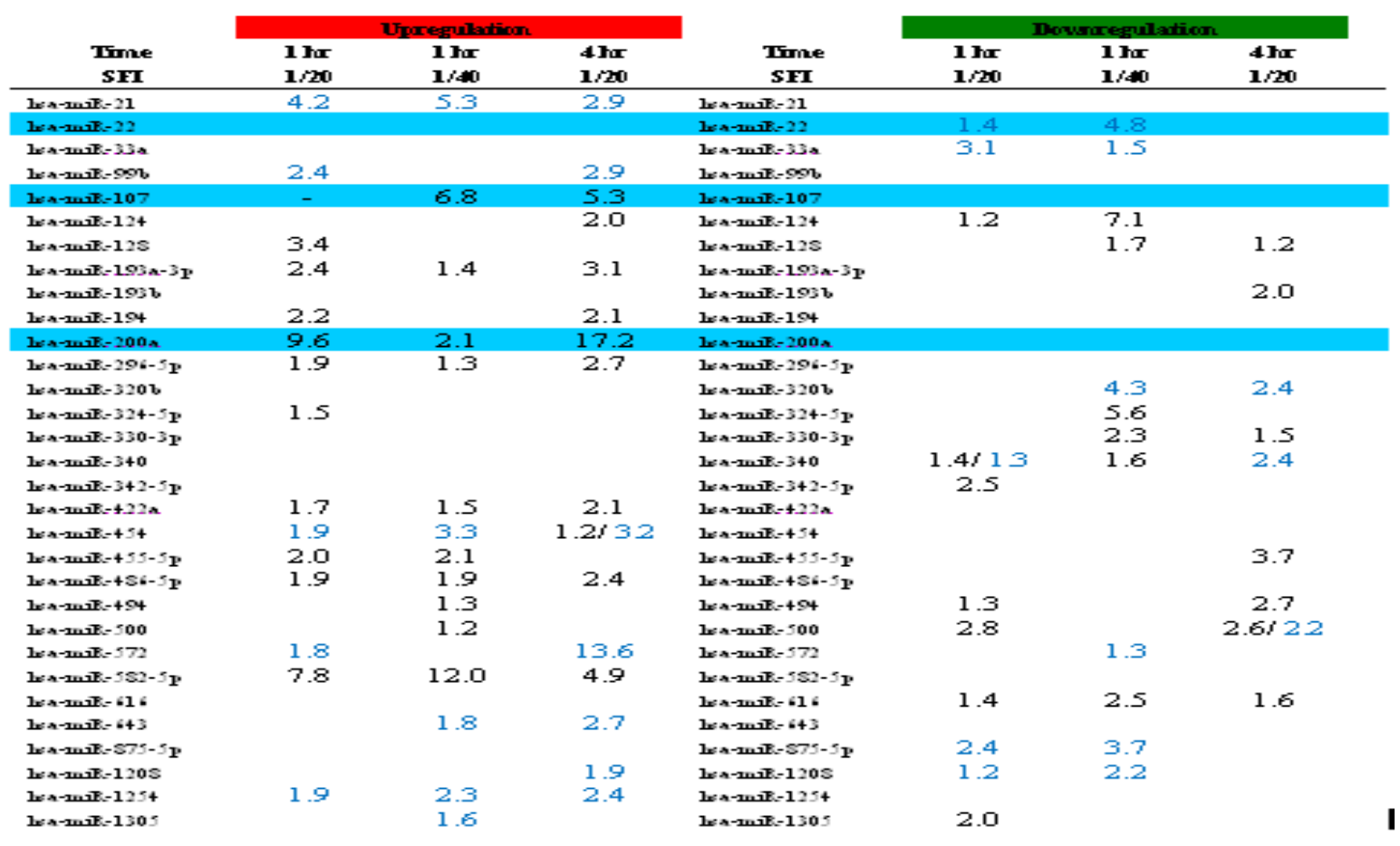

Figure 1: 17 miRNAs showed upregulated and 16 miRNAs were downregulated as listed among which miR-22 demonstrated significant downregulation by 4.8 fold under the treatment of 1 in 40 diluted SFI for 1 hour; miR-107 was significantly upregulated by 6.8 fold and 5.3 fold in the treatment of 1 in 40 diluted SFI for 1 hour and 1 in 20 diluted SFI for 4 hours respectively. Further, miR-200a was also significantly upregulated by 9.6 and 17.2 fold in the treatment with 1 in 20 diluted SFI for 1 hour and 4 hours respectively. Adding the computational evidence of miR-22, miR-107 and miR-200a in association with gene CD80, these three miRNAs were suggested to be involved in the costimulatory regulation on the expression of CD80 over the THP-1 cells and probably the MDDCs treated with SFI through the activation on the TLR-4 receptor.

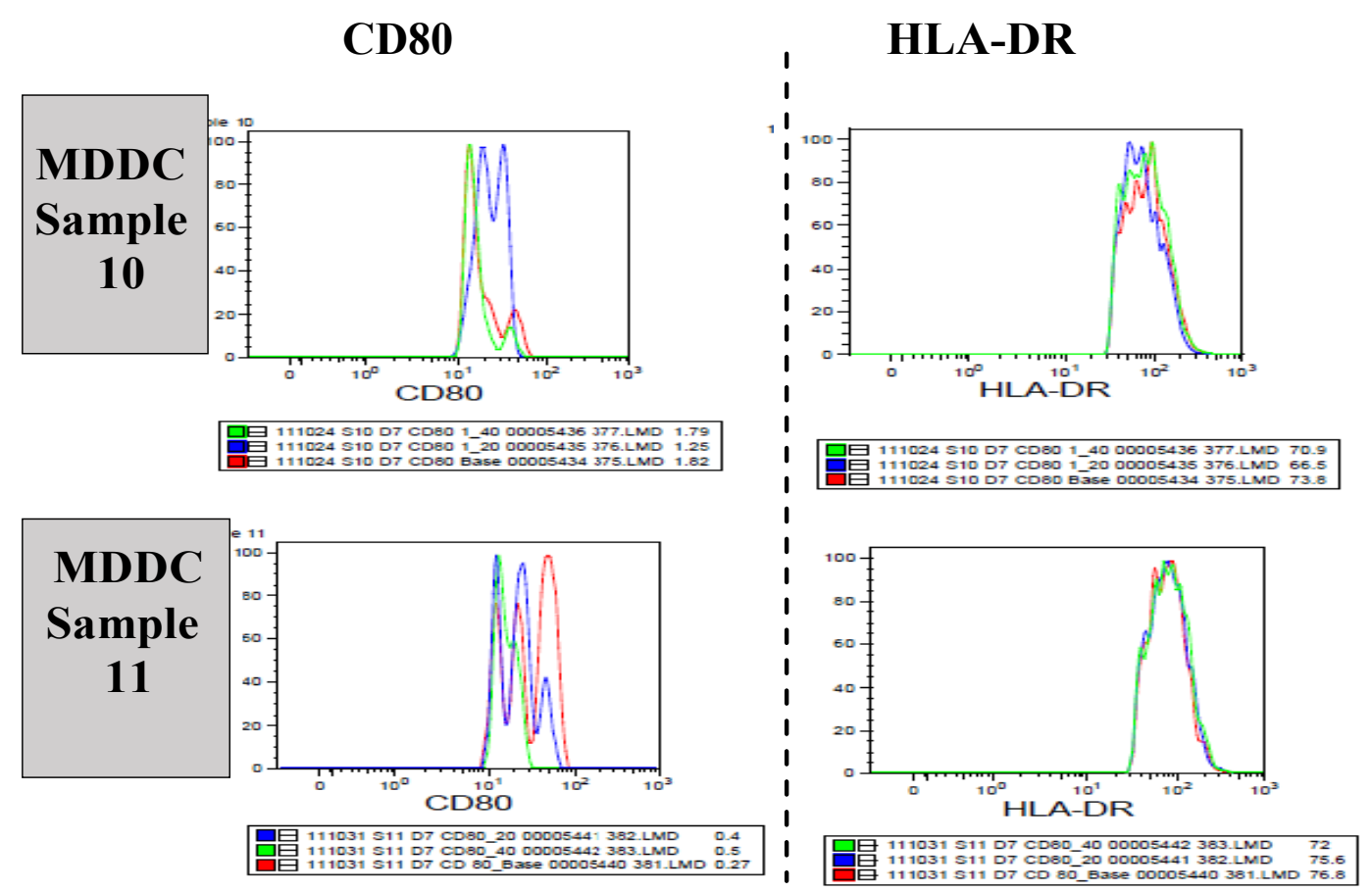

Figure 2: Three histograms of expression on each cell markers of CD80 and HLA-DR for each of the MDDC Sample 10 and MDDC Sample 11 were displayed as exemplars. Histogram in red line showed the cell marker profile of MDDCs without SFI treatment. These saline controls served as the baseline for the comparison with two other histograms: blue (SFI diluted 1 in 20) and green (SFI diluted 1 in 40) accordingly. 


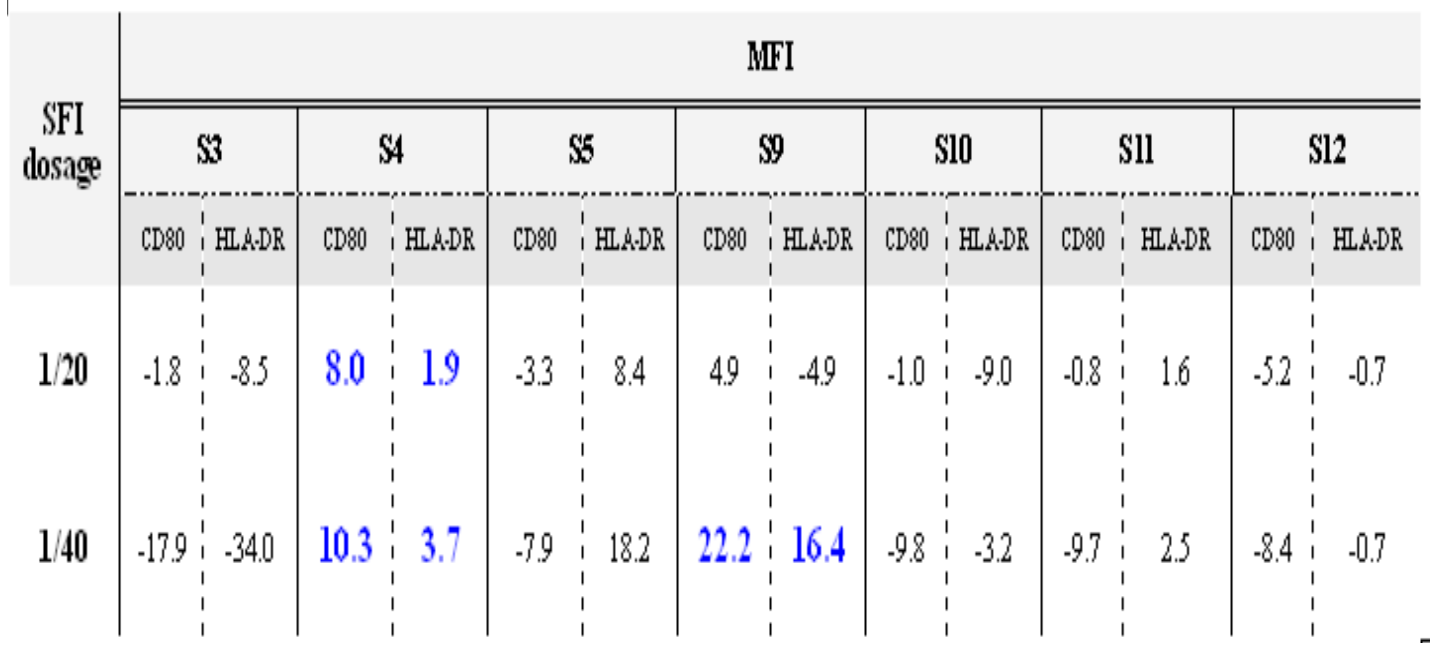

Figure 3: Up-regulated expression change of CD80 was observed on samples $S 4$ and $S 9$ in SFI dosages of $1 / 20$ \& $1 / 40$. For expression change of HLA-DR, upregulation of the marker was seen on S4 in SFI of $1 / 20$ \& $1 / 40$ and S9 in SFI of 1/40 respectively. Out of the eight MDDC samples S2 to S5 and S9 to S12, only S4 and S9 were activated by SFI in the experiments.

miRNAs were possibly the target miRNAs having the capability by means of in epigenetic activities to influence the expression of co-stimulatory cell marker CD80 on the immune cells, on the basis of searches in the database pipelines, PicTar and miRanda-mirSVR [5]. The seed region at the 5'_uAACACUGucugguaacgaugu_3' of hsa-miR-200a may crossover and act on the complimentary 3'_aUUGUGACcauaagagauuuca_5' at 250 base pair region of the CD80 molecules. Similarly, the 5' aAGCUGCCAGUUGAAGaacugu_3' region of hsa-miR-22 and the 5'_aGCAGCAUuguacagggcuauca_3' of hsa-miR-107 may align complementarily with the 3'_aUCGACGGUACUCUACacguaa_5' at 1162 base pair region and the 3'_uCGUCGUAuuuuagaagaugaga_5' at around 487 base pair of the CD80 molecules respectively.

\section{Results}

In the results of the miRNA array study, it was found that there were a number of miRNAs showing expression ( $\geqq 1.2$ fold) which had been experimentally verified and could be matched with the genes in the TLR-4 related signaling pathways including My D88 dependent, MyD88 independent and MHCII. The positive results with the miRNA expression were further processed and filtered by focusing on those miRNAs with greater fold change ( $\geqq 2$ ) and probable association with the costimulatory CD80 gene (Figure 1). Adding the computational evidence of miR-22, miR-107 and miR-200a in association with gene CD80, these three miRNAs were suggested to be involved in the costimulatory regulation on the expression of CD80 over the THP-1 cells and probably the MDDCs treated with SFI through the activation on the TLR-4 receptor.

The expression the costimulatory molecule CD80 and HLA-DR on MDDCs with or without SFI were analyzed after 24 hours incubation. Figure 2 illustrated the three histograms of expression on each cell markers of CD80 and HLA-DR for the MDDC samples S10 \& S11 as exemplars. The flow cytometric analysis of the cell markers CD80 and HLA-DR was performed on the seven SFI treated MDDC samples. The results of the expression change of the CD80 and HLA-DR were illustrated (Figure 3).

There were some positive confirmatory results observed over the expression changes of the cell markers and miRNAs on samples $S 4$ and
S9 although the statistical significance of the test verification in Figure 4 was not demonstrated. The up-regulation of CD80 and HLA-DR of sample S4 (Figure 3) were observed in both SFI dosages of $1 / 20$ and $1 / 40$ showing the MDDCs of S4 had been activated after 24 hours incubation while the miR-107 and miR-200a were also up-regulated in both short time points of 1 hour and 4 hours (Figure 5).

These results demonstrated individual MDDC sample would be activated by the SFI similar to that observed in THP-1 cell model. Another MDDC sample S9 showed up-regulated CD80 and HLA-DR in SFI of $1 / 40$ while miR-22 was down-regulated significantly in 1 hour time point under the same SFI dosage, which illustrated the similar test result pattern in THP-1 cells.

Concerning the positive validated results of the target miRNAs which were matched with that in the miRNA array study in using the THP-1 cell model, the up-regulated miR-107 expression was observed in S2, S4 and S10 to S12 at 1/40 SFI in 1 hour time. miR-107 and miR200a were up-regulated in 5 out of 7 MDDC samples, S3 to S5 \& S10 to S11, at $1 / 20$ SFI dosage in 4 hours time and $1 / 40$ SFI in 1 hour time respectively. Another MDDC sample $S 9$ showed up-regulated CD80 and HLA-DR in SFI dosage of 1/40 meanwhile miR-22 was down-regulated significantly in 1 hour time point under the same SFI dosage, which illustrated the similar test result pattern in THP-1 cells. Furthermore, miR-22 showed down-regulation in half of the MDDC samples including S2, S3, S5 and S9 under the SFI dosage of $1 / 40$ in 1 hour time.

\section{Statistical analysis}

The results of the miRNA expression levels in the eight MDDC samples treated with SFI and of the cell marker expression levels in the seven MDDC samples treated with SFI were analysed. Pearson's product moment correlation was used to verify whether the expression changes of surface marker CD80 and HLA-DR correlated with the fold change expression of the three target miRNAs under the effects of SFI treatment by the paired sample t-test. One way ANOVA was applied to determine if there were statistically significant differences between sample groups in expression levels of three miRNAs and the two cell markers. All statistical analyses were performed by using SPSS 
Citation: Yuwh H, Sze WH, Yip DMY, Cho SP, Boost WCS, et al. (2014) To Study the Effects of Shenqifuzheng Injection (SFI) on Micro-RNAs in Human Dendritic Cells. J Data Mining Genomics Proteomics 5: 149. doi:10.4172/2153-0602.1000149

\begin{tabular}{|c|c|c|c|c|c|c|c|c|c|c|}
\hline $\operatorname{miRNA}$ & & Period & $\mathrm{S} 2$ & S3 & S4 & S5 & S9 & $\mathrm{S} 10$ & S11 & $\mathrm{S} 12$ \\
\hline \multirow{3}{*}{$\operatorname{miR}-22$} & \multirow{2}{*}{$\mathrm{SFI} 1 / 20$} & $1 \mathrm{hr}$ & 0.3 & 1.0 & 1.1 & 0.2 & 0.13 & 1.0 & 2.4 & 1.2 \\
\hline & & $4 \mathrm{hr}$ & & 0.9 & 1.3 & 1.0 & 1.0 & 2.9 & 4.0 & 1.5 \\
\hline & SFI 1/40 & $1 \mathrm{hr}$ & 0.8 & 0.5 & 1.0 & 0.8 & 0.17 & 1.2 & 1.9 & 1.1 \\
\hline
\end{tabular}

\begin{tabular}{|c|c|c|c|c|c|c|c|c|c|c|}
\hline \multirow[b]{2}{*}{$\operatorname{miR}-107$} & $\mathrm{SFI} 1 / 20$ & $\frac{1 \mathrm{hr}}{4 \mathrm{hr}}$ & 1.6 & $\begin{array}{l}0.8 \\
0.6\end{array}$ & $\begin{array}{l}1.2 \\
1.2\end{array}$ & $\begin{array}{c}0.14 \\
0.8\end{array}$ & $\begin{array}{c}0.09 \\
0.8\end{array}$ & $\begin{array}{l}1.0 \\
1.8\end{array}$ & $\begin{array}{l}1.5 \\
21\end{array}$ & $\begin{array}{l}1.5 \\
1.9\end{array}$ \\
\hline & $\mathrm{SFI} 1 / 40$ & $1 \mathrm{hr}$ & 1.1 & 0.4 & 1.1 & 0.6 & 0.08 & 1.5 & 1.1 & 1.2 \\
\hline \multirow{3}{*}{ miR:2010a } & $\mathrm{SFI} 1 / 20$ & $1 \mathrm{hr}$ & 0.4 & 1.2 & 1.4 & 0.16 & 0.13 & 1.2 & 0.9 & 0.62 \\
\hline & & $4 \mathrm{hr}$ & & 1.1 & 1.6 & 1.1 & 0.4 & 3.2 & 2.1 & 0.8 \\
\hline & SFI 1/40 & $1 \mathrm{hr}$ & 1.0 & 0.8 & 1.0 & 0.7 & 0.07 & 1.5 & 0.9 & 0.56 \\
\hline
\end{tabular}

Figure 4: Fold change of miRNAs of samples $S 2$ to S5 \& S9 to S12 in diluted SFI at two time points.

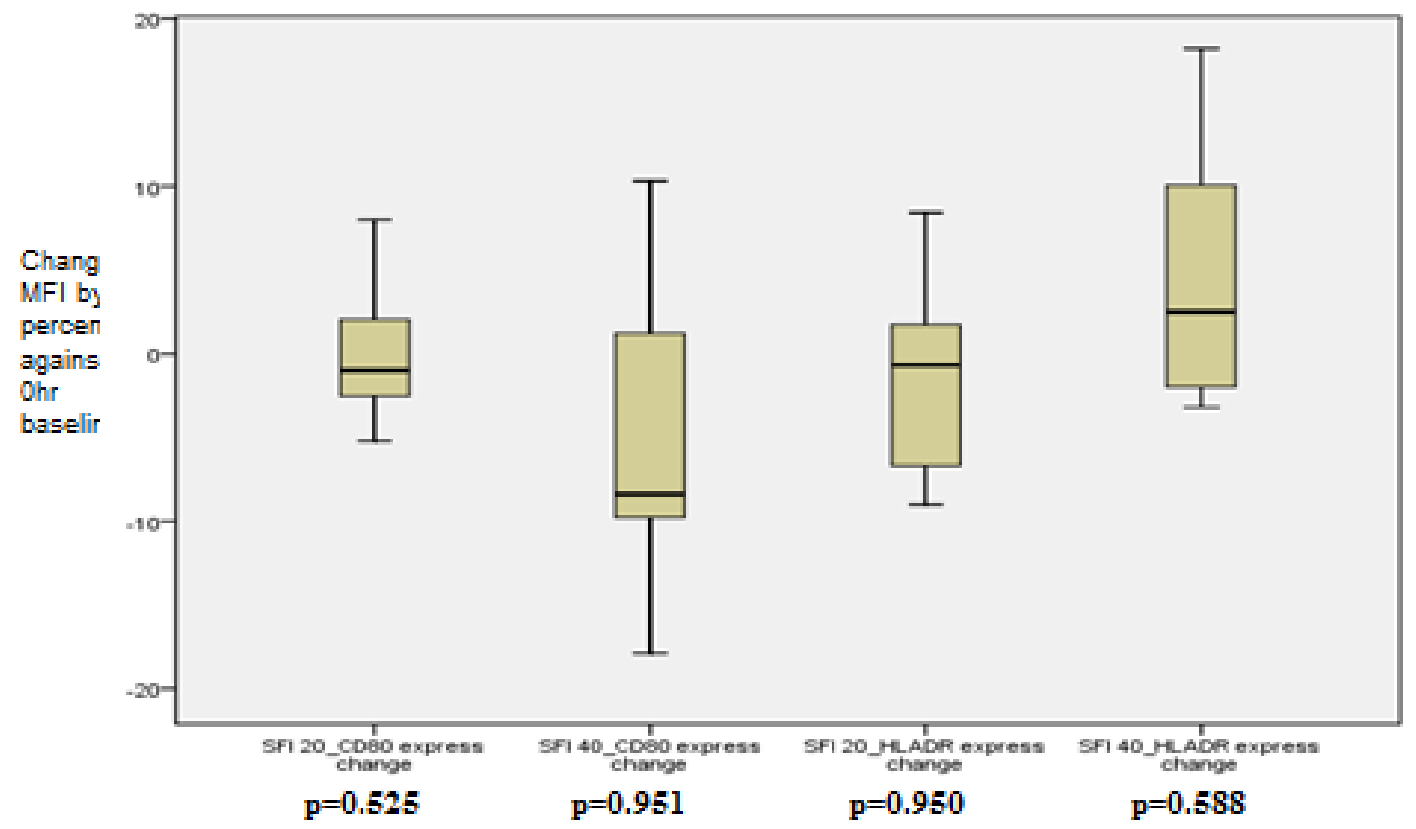

Figure 5: Box plots on expression of CD80 and HLA-DR on MDDCs treated with SFI of $1 / 20$ and $1 / 40$ in dilution over $24 \mathrm{hrs}$ incubation. The results of expression changes of CD80 and HLA-DR over seven MDDC samples ( $p>0.05$ ) treated with SFI 1/20 and SFI 1/40 against their own saline controls after $24 \mathrm{hrs}$ incubation showed that the expression change of the two cell markers were statistically significant.

(version 17.0) software (SPSS Inc., USA). $\mathrm{P}<0.05$ was considered to be significant.

\section{Discussion}

The miRNA array studies involving the two SFI exposures of $1 / 20$ and $1 / 40$ provided a number of positive findings involving interacting
miRNAs which were up-regulated or down-regulated at the times of $1 \mathrm{~h}$ and $4 \mathrm{~h}$. Over $50 \%$ or 200 miRNAs out of the 384 miRNAs in the array plates A \& B demonstrated up-regulation or down-regulation with fold change greater or equal to 1.2 , which demonstrates the extremely wide range of effects on miRNA activities present in the SFI treated THP1 cells which may be related to the signaling reception of TLR-4. The results of this study were not as expected and differed from those of 


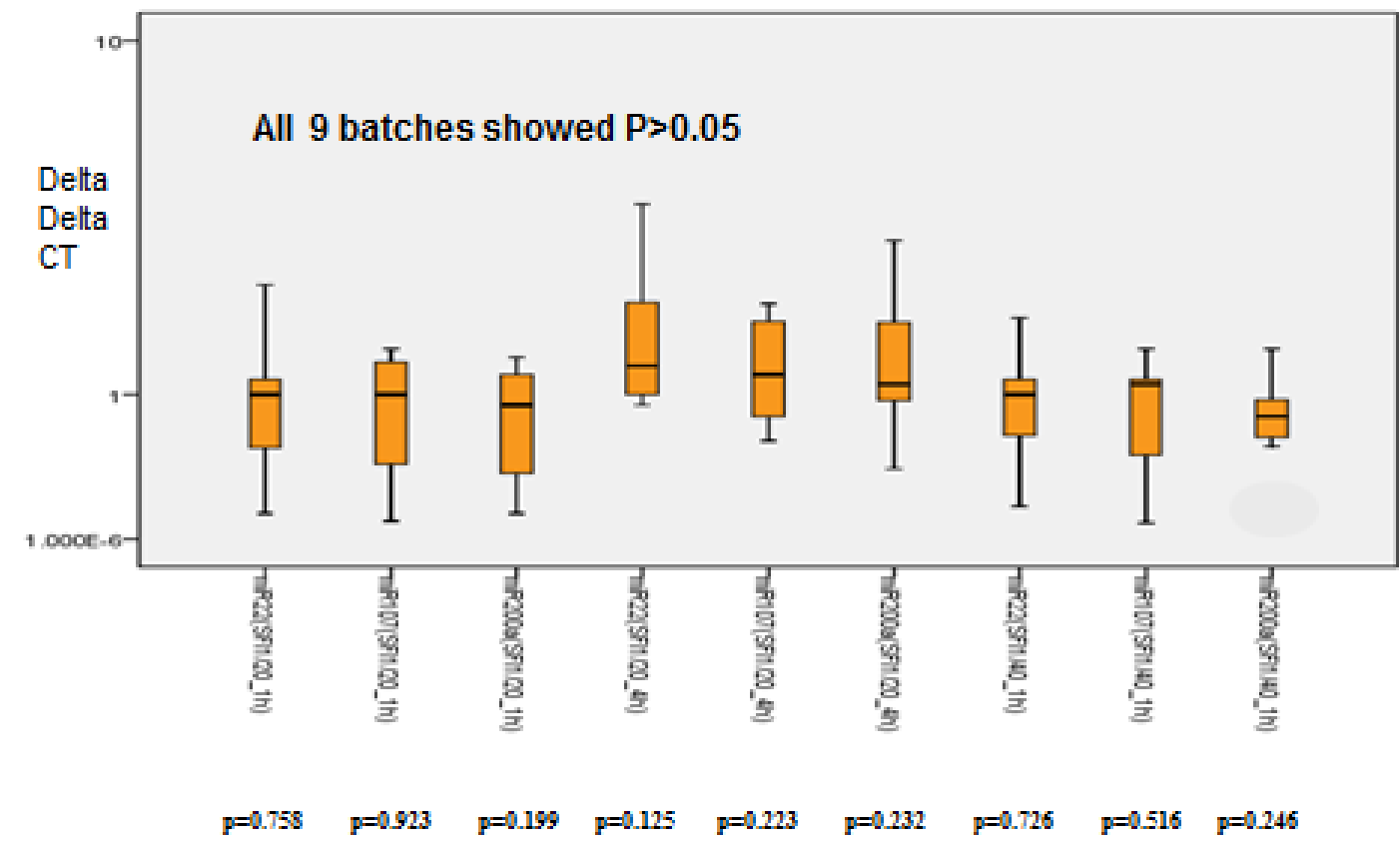

Figure 6: Box plots on the miRNAs differential expression in MDDC samples. The results of miRNAs expression change over the eight MDDC samples against their buffer saline base lines showed that the fold changes of all three miRNAs $(p>0.05)$ under differential SFI treatments were statistically insignificant.

previously reported studies in which LPS was used to stimulate THP-1 cells initiating over expression of miR-146a [11-15], miR-132 or miR155 [16] reported an unique pattern of gradual increase of miR-146a starting $4 \mathrm{~h}$ after LPS stimulation in THP-1 cells, continuing up to 35fold over $24 \mathrm{~h}$ [17] examined the effect of LPS on miR-146a using THP-1 cells and found the expression level of miR-146a increased from 19 fold up to 100 fold during LPS stimulation. The current study reproduced the up-regulation of miR-146a from 4 to 10 fold by stimulating THP-1 cells using the LPS when it was used as a positive control setup in the pilot study in optimization of SFI exposure and incubation time. Method of miRNA array used in this study may account for the unexpected observation of miRNA expression outcome from that observed in the previous studies with quantification directly using real time RT-PCR. From the observation of miRNAs having a higher degree of fold change expression $\geqq 2$, over 20 miRNAs were recognized of which miR-22, miR-107 and miR-200a were then identified in the association with the computational evidence on the CD80 gene [18] although there was no other evidence or previous studies on these miRNAs in linking them with DCs. They were selected to be the target miRNAs for subsequent confirmatory studies in the MDDC samples.

At the time of this study, there was no little information about miR-22, miR-107 and miR-200a in association with DCs available. A positive correlation between MGC14376 and miR-22 was reported in the microarray analysis for U937 monocytic differentiation $[19,20]$. Li et al. (2010) [14] proposed that the accumulation of marker H3K27 trimethylation may be a novel epigenetic mechanism for miR-22 silencing in acute lymphoblastic leukemia. It was suggested that miR-22 may target Irf8 mRNA for posttranscriptional repression and have influential effects on DC subset differentiation. Differential over-expression of miR-107 in the treated human cancer cell lines, MiaPACA-2 and PANC1 by a demethylating agent was observed and it was associated with the target gene, cyclin-dependent kinase 6 providing a functional basis for the epigenetic inactivation of this miR-107 in pancreatic cancer [19].
miR-200a over-expression reduces a number of cancers such as prostate cancer [4], breast cancer [18-22] and ovarian cancer [23]. Some positive confirmatory results expression changes of the cell markers and miRNAs on samples $\mathrm{S} 4$ and $\mathrm{S} 9$ were observed but analysis of differences for the group revealed there was no significant difference overall. Up-regulation of CD80 and HLA-DR of sample S4 were observed in exposure to both $1 / 20$ SFI and 1/40 SFI showing the MDDCs of S4 had already been activated after $24 \mathrm{~h}$ incubation. miR-107 was also upregulated after both short exposure times of $1 \mathrm{~h}$ at $1 / 40$ SFI and $4 \mathrm{~h}$ at $1 / 20$ SFI and the miR-200a after exposure of both SFI concentrations for 1 hour (Figure 6). The up-regulation of miR-107 and miR-200a in sample 44 exactly matched the results obtained in using the THP- 1 cell model, which could miR-200a were up-regulated in 5/7 MDDC samples (S3 to S5 \& S10 to S11) at 1/20 SFI. As DC populations vary between individuals, a larger sample size may reveal a significant effect among a sub-group of subjects for up-regulation of these parameters. This genetic variation may be another determinant for the outcome observed: only a proportion of the MDDC samples showed a positive response to SFI in over expression of miR-107 and miR-200a because Irf8 mRNA for posttranscriptional repression and have influential effects on DC subset certain of the sub-populations of DC may be genetically specific for the immune-modulatory effect of SFI. Further studies on the relationship between the sub-types of DCs and their specific responses to SFI may allow the effective therapeutic use of this TCM drug to be targeted for certain patients in a population. SFI has already been demonstrated with its effectiveness of improvement in clinical symptoms and life quality of the cancer patients [20], it may be necessary to determine which patients are most likely to benefit. With respect to the positive validated results of the target miRNAs which were matched with those of the miRNA array study using the THP-1 cell model, up-regulated miR-107 expression was observed in S2, S4 and S10 to S12 at 1/40 SFI after $1 \mathrm{~h}$. miR-107 and miR-200a were up-regulated in 5/7 MDDC samples (S3 to S5 \& S10 to S11) at 1/20 SFI exposure for $4 \mathrm{~h}$ and $1 / 40$ SFI for $1 \mathrm{~h}$. Sharma, et al. [24] evaluated the clinical significance of 
Citation: Yuwh H, Sze WH, Yip DMY, Cho SP, Boost WCS, et al. (2014) To Study the Effects of Shenqifuzheng Injection (SFI) on Micro-RNAs in Human Dendritic Cells. J Data Mining Genomics Proteomics 5: 149. doi:10.4172/2153-0602.1000149

miR-107 expression in esophageal squamous cell carcinoma (ESCC) patients and discovered that significant down-regulation of miR-107 was observed in neoplastic and preneoplastic tissues. Investigation of the role of miR-107 in tumorgenesis demonstrated that p53-induced miR-107 suppresses brain tumour cell growth and down-regulates CDK6 and NOTCH2 expression. These findings support the tumour suppressor role of miR-107 as a target for glioma therapy [25]. This suggested that SFI is potentially therapeutically useful in the regulation of miR-107 in the ESCC and brain tumour patients. Manavalan, et al. [18] reported expression of miR-200a was lowered in LY2 endocrineresistant mesenchymal breast cancer cells. Expression levels of miR200a were also reported to be significantly down-regulated in latestage (FIGO III+V) and grade 3 groups as compared with early stage (FIGO I+II) and grade 1 to 2 groups [23] suggesting miR-200a is a candidate target for the development of new treatment modelities against ovarian cancer. Su et al., [26] demonstrated that miR-200a acts as a tumour suppressor, regulating the activity of the $\mathrm{Wnt} / \beta$-catenin signaling pathway, which is involved in tumour progression of many cancers. Thus, SFI is potentially useful in the treatments of various cancers. The effects of increased expression levels of miR-107 and miR200a in link with the therapeutic effects of SFI are probably related to their association with the CD80 gene. Further studies to improve our understanding of the modulatory mechanisms of the immune system by SFI would be worthwhile in determining the use of this agent in therapy.

Another MDDC sample S9 showed up-regulation of CD80 and HLA-DR in 1/40 SFI while miR-22 was significantly down-regulated at the same concentration for $1 \mathrm{~h}$. These also paralleled results observed using the THP-1 cells. Furthermore, miR-22 showed down-regulation in half of the MDDC samples following exposure of 1/40 SFI for $1 \mathrm{~h}$. Teiten et al. [27] postulated that the polyphenolic compound curcumin (diferuloyl methane) may modulate some miRNAs including miR-22 and then their multiple target genes. Epigenetic regulation balance may act as a preventive or therapeutic agent against human cancer. Over-expression of miR-22 has been shown to induce cardiomyocyte hypertrophy $[11,27]$. Significant down-regulation of miR-22 by SFI may assist in the regulation of cardiac hypertrophy and remodeling in response to stress. This study of the immune-modulatory effects of SFI, whilst not demonstrating uniform effects on DC cells, does demonstrate that the agent does have beneficial effects, even if some of these may be restricted to certain sub-groups of the population. However, this was a small scale study and certain limitations of the techniques used must be considered.

\section{References}

1. Ardavín C, Amigorena S, Reis e Sousa C (2004) Dendritic cells: immunobiology and cancer immunotherapy. Immunity 20: 17-23.

2. Auffray C, Chen Z, Hood L, Soares B, Sugano S (2003) Foreword: from the TRANSCRIPTOME conferences to the SYSTEMOSCOPE international consortium. C R Biol 326: 867-875.

3. Auffray C, Chen Z, Hood L (2009) Review systems medicine: the system of medical genomics and health care. Genome Med 1:2

4. Barron N, Keenan J, Gammell P, Martinez VG, Freeman A, et al. (2012) Biochemical relapse following radical prostatectomy and miR-200a levels in prostate cancer. Prostate 72: 1193-1199.

5. Betel D, Koppal A, Agius P, Sander C, Leslie C (2010) Comprehensive modeling of microRNA targets predicts functional non-conserved and non-canonical sites. Genome Biol 11: R90.

6. Borghaei H, Smith MR, Campbell KS (2009) Immunotherapy of cancer. Eur J Pharmacol 625: 41-54.
7. Chen L, Zhang R, Li P, Liu Y, Qin K, et al. (2013) P53-induced microRNA-107 inhibits proliferation of glioma cells and down-regulates the expression of CDK6 and Notch-2. Neurosci Lett 534: 327-332.

8. Dai Z, Wan X, Kang H, Ji Z, Liu L, et al. (2008) Clinical effects of shenqi fuzheng injection in the neoadjuvant chemotherapy for local advanced breast cancer and the effects on T-lymphocyte subsets. J Tradit Chin Med 28: 34-38.

9. Gao SR, Xia HP (2009) Effect of Shenqifuzheng injection combined with chemotherapy in treatment of advanced breast cancer on T-lymphocyte AgNORs. Chinese Clinical Oncol 14: 1003-1006.

10. Holmstrøm K, Pedersen AW, Claesson MH, Zocca MB, Jensen SS (2010) Identification of a microRNA signature in dendritic cell vaccines for cancer immunotherapy. Hum Immunol 71: 67-73.

11. Huang ZF, Wei JS, Li HZ (2008) [Effect of Shenqi Fuzheng injection combined with chemotherapy on thirty patients with advanced breast cancer]. Zhongguo Zhong Xi Yi Jie He Za Zhi 28: 152-154.

12. Jefford M, Schnurr M, Toy T, Masterman KA, Shin A, et al. (2003) Functional comparison of DCs generated in vivo with Flt3 ligand or in vitro from blood monocytes: differential regulation of function by specific classes of physiologic stimuli. Blood 102: 1753-1763.

13. Lee KH, Lotterman C, Karikari C, Omura N, Feldmann G, et al. (2009) Epigenetic silencing of MicroRNA miR-107 regulates cyclin-dependent kinase 6 expression in pancreatic cancer. Pancreatology 9: 293-301.

14. Li X, Liu J, Zhou R, Huang S, Huang S, et al. (2010) Gene silencing of MIR22 in acute lymphoblastic leukaemia involves histone modifications independent of promoter DNA methylation. Br J Haematol 148: 69-79.

15. Li HS, Greeley N, Sugimoto N, Liu YJ, Watowich SS (2012) miR-22 controls Irf8 mRNA abundance and murine dendritic cell development. PLoS One 7 e52341.

16. Lin A, Schildknecht A, Nguyen LT, Ohashi PS (2010) Dendritic cells integrate signals from the tumor microenvironment to modulate immunity and tumor growth. Immunol Lett 127: 77-84.

17. Lin HS, Li DR (2007) [Multi-center randomized clinical study on Shenqi-fuzheng injection combined with chemotherapy in the treatment for lung cancer] Zhonghua Zhong Liu Za Zhi 29: 931-934.

18. Manavalan TT, Teng Y, Litchfield LM, Muluhngwi P, Al-Rayyan N, et al. (2013) Reduced expression of miR-200 family members contributes to antiestrogen resistance in LY2 human breast cancer cells. PLoS One 8: e62334.

19. Nahid MA, Pauley KM, Satoh M, Chan EK (2009) miR-146a is critical for endotoxin-induced tolerance: IMPLICATION IN INNATE IMMUNITY. J Bio Chem 284: 34590-34599.

20. Pauley KM, Satoh M, Pauley BA, Dominguez-Gutierrez PR, Wallet SM, et al. (2010) Formation of GW/P bodies as marker for microRNA-mediated regulation of innate immune signaling in THP-1 cells. Immunol Cell Biol 88: 205-212.

21. Schmelzer C, Kitano M, Rimbach G, Niklowitz P, Menke T, et al. (2009) Effects of ubiquinol-10 on microRNA-146a expression in vitro and in vivo. Mediators Inflamm 2009: 415437

22. Sender LY, Gibbert K, Suezer Y, Radeke HH, Kalinke U, et al. (2010) CD40 ligand-triggered human dendritic cells mount interleukin-23 responses that are further enhanced by danger signals. Mol Immunol 47: 1255-1261.

23. Xu S, Xu P, Wu W, Ou Y, Xu J, et al. (2013) The biphasic expression pattern of miR-200a and E-cadherin in epithelial ovarian cancer and its correlation with clinicopathological features. Curr Pharm Des

24. Sharma P, Saraya A, Gupta P, Sharma R (2013) Decreased levels of circulating and tissue miR-107 in human esophageal cancer. Biomarkers 18: 322-330.

25. Steinman RM (2008) Dendritic cells in vivo: a key target for a new vaccine science. Immunity 29: 319-324.

26. Su J, Zhang A, Shi Z, Ma F, Pu P, et al. (2012) MicroRNA-200a suppresses the Wnt $/ /^{2}$-catenin signaling pathway by interacting with $\hat{I}^{2}$-catenin. Int $\mathrm{J}$ Oncol 40 : 1162-1170.

27. Teiten MH, Dicato M, Diederich M (2013) Curcumin as a regulator of epigenetic events. Mol Nutr Food Res 57: 1619-1629. 